\title{
LncRNA DIx6os1 Accelerates Diabetic Nephropathy Progression by Epigenetically Repressing SOX6 via Recruiting EZH2
}

\author{
Yan-Xia Chen Shu-Ying Zhu Chong Huang Cheng-Yun Xu \\ Xiang-Dong Fang Wei-Ping Tu \\ Department of Nephrology, The Second Affiliated Hospital of Nanchang University, Nanchang, PR China
}

\author{
Keywords \\ LncRNA Dlx6os1 · Diabetic nephropathy · Enhancer of zeste \\ homolog $2 \cdot$ SOX6
}

\begin{abstract}
Introduction: Diabetic nephropathy (DN) is the leading cause of kidney failure worldwide. To explore the pathogenesis and effective biological target of DN is beneficial to seeking novel treatment strategies. Objective: This study aimed to investigate the role of the IncRNA DIx6os1/SOX6/EZH2 axis in DN progression. Methods: PAS staining was performed to evaluate extracellular matrix accumulation; ELISA was carried out to assess the levels of urine microalbumin and blood glucose concentration; RT-qPCR was carried out to detect the levels of IncRNA DIx6os1, TNF-a, IL-1 $\beta$, IL-6, SOX6, and EZH2. Western blot was performed to assess the levels of Col-IV, FN, TGF- $\beta 1$, and SOX6 proteins. RIP assay was carried out to verify the interaction between IncRNA DIx6os 1 and EZH2. ChIP-qPCR was conducted to verify the interaction between EZH2 and SOX6 promoter. Results: Our results illustrated that IncRNA DIx6os1 was highly expressed in DN mice and HG-induced SV40 MES13 cells. LncRNA Dlx6os1 knockdown inhibited HG-induced SV40 MES13 cell proliferation, fibrosis, and inflammatory cytokine release. LncRNA Dlx6os 1 inhibited SOX6 expression by recruiting EZH2 in HGSV40 MES13 cells, and SOX6 mediated the effects of IncRNA
\end{abstract}

Dlx6os1 on proliferation, fibrosis, and inflammatory factor release of HG-induced SV40 MES13 cells. Conclusion: LncRNA DIx6os1 accelerates the progression of DN by epigenetically repressing SOX6 via recruiting EZH2.

(c) 2022 The Author(s).

Published by S. Karger AG, Basel

\section{Introduction}

Diabetic nephropathy (DN) is a progressive kidney disease caused by diabetes, which activates a variety of classical regeneration pathways [1-3]. The progression of $\mathrm{DN}$ is a long process, and its early symptoms are not obvious, while its late symptoms are characterized by increased infiltration of inflammatory cells, globular sclerosis, thickening of the subterranean membrane, and tubular atrophy, which eventually lead to renal failure $[4,5]$. Therefore, figuring out the pathogenesis of DN to looking for early biomarkers is beneficial to the prevention and treatment of DN.

Long noncoding RNA (lncRNA) is a noncoding RNA with a length of more than 200 nucleotides that lacks the ability to encode proteins and interact directly with a variety of RNA molecules or proteins [6]. Increasing evidence shows that lncRNA regulates various bioactivities by regulating target genes' expression at transcriptional karger@karger.com www.karger.com/kbr

Karger $\stackrel{\text { ' }}{5}$

GOPEN ACCESS
(C) 2022 The Author(s)

Published by S. Karger AG, Basel

This is an Open Access article licensed under the Creative Commons Attribution-NonCommercial-4.0 International License (CC BY-NC) (http://www.karger.com/Services/OpenAccessLicense), applicable to the online version of the article only. Usage and distribution for commercial purposes requires written permission.
Correspondence to:

Wei-Ping Tu, tuweiping6102@sina.com 
and posttranscriptional levels [7]. LncRNA has been shown to be involved in the development of a variety of diseases, including cancer, diabetes, DN, and inflammatory diseases $[5,8,9]$. For example, lncRNA NEAT1 accelerates DN progression by sponging miR-23c [10]. Furthermore, Cheng et al. [11] reported that IncRNA Dlx6os1 serves as a potential therapeutic target of $\mathrm{DN}$ to regulate inflammation development. Chen et al. [12] revealed that lncRNA Dlx6os1 knockdown inhibits SV40 MES13 cell proliferation and fibrosis and accelerates cell apoptosis. However, the specific mechanism by which lncRNA Dlx6os1 regulates DN has not been fully explained.

SOX protein is a transcription factor with high motor DNA binding domain, which is involved in target genes' regulation by binding to them in the promoter region [13]. SRY-related high-mobility-group box 6 (SOX6) belongs to the SOX family, which has been shown to be involved in various biological processes [14]. Li et al. [15] revealed that SOX6 interacts with netrin-1 to regulate the metastasis and angiogenesis of ovarian cancer cells. It has been reported that SOX6 is also involved in the regulation of kidney disease and cardiovascular disease [16]. However, the specific role of SOX6 in DN has not been reported.

In summary, the purpose of this study is to clarify the regulatory role of lncRNA Dlx6os 1 in DN and the possible mechanism for SOX6 to regulate DN progression. We proved that $\operatorname{lncRNA}$ Dlx6os 1 accelerates proliferation, fibrosis, and inflammatory cytokine release of SV 40 MES13 cells by epigenetically repressing SOX6 via recruiting enhancer of zeste homolog 2 (EZH2). Our study figured out the role of lncRNA Dlx6os1 in $\mathrm{DN}$ and provides new insights into the development of DN treatment strategies.

\section{Materials and Methods}

\section{Animal}

Male mice (C57BL/KSJ-db/db) and normal-born mice $(\mathrm{db} / \mathrm{m})$ were supplied by Shanghai Sippr-BK Experimental Animals Co., Ltd. (Shanghai, China). The mice were kept in cages in airy rooms and provided with plenty of food and water. This study protocol was reviewed and the need for approval was waived by The Second Affiliated Hospital of Nanchang University. For each particular genotype, male mice, 8 weeks old, were randomly divided into 2 subgroups (each having at least 6 mice): $\mathrm{db} / \mathrm{db}$ mice were administered a single intraperitoneal injection of STZ $(200 \mathrm{mg} / \mathrm{kg})$, and $\mathrm{db} / \mathrm{m}$ mice were injected with citrate buffer. Immediately after the diabetic induction, blood glucose was assayed with a Glucometer (OneTouch Ultra; LifeScan, Milpitas, CA, USA), and only those mice with blood glucose levels above $16 \mathrm{mmol} / \mathrm{L}$ were considered to be diabetic. All the $\mathrm{db} / \mathrm{db}$ or $\mathrm{db} / \mathrm{m}$ mice were euthanized at 24 weeks of age.

\section{Cell Culture and DN Cell Model}

SV40 MES13 cells were purchased from Procell Life Technology Co., Ltd. (Wuhan, China). Cells were cultured in Dulbecco modified Eagle's medium (Roche, Basel, Switzerland) containing $1 \%$ penicillin streptomycin solution and $10 \%$ fetal bovine serum (Solarbio, Beijing, China). All cells were cultured in a wet incubator containing $5 \% \mathrm{CO}_{2}$ at $37^{\circ} \mathrm{C}$. SV $40 \mathrm{MES} 13$ cells were incubated for $24 \mathrm{~h}$ with $25 \mathrm{mM}$ glucose (HG) to establish a DN cell model, and compared cells were cultured with $5.5 \mathrm{~mm}$ glucose (NG).

\section{PAS Staining}

Specimens were fixed for 10 min with $10 \%$ ethanol and embedded with OCT or paraffin. The slice was cut into $7-\mu \mathrm{m}$-thick slices, rinsed for $10 \mathrm{~s}$ with PBS, oxidized for $10 \mathrm{~min}$ with $1 \%$ high iodine acid solution, rinsed for $10 \mathrm{~s}$ with PBS, dried using filter paper, and kept at room temperature to air dry. Slices were then soaked with Schiff reagents for $30 \mathrm{~min}$ (abris-plus, $4^{\circ} \mathrm{C}$ preservation) and then underwent PBS wash $5 \mathrm{~min}$, hematoxylin re-dye $5 \mathrm{~min}$, and PBS rinsing.

\section{Cell Transfection}

The transfection doses for si-lncRNA Dlx6os1, si-EZH2, and si-SOX6 and its negative control si-NC (synthesized by Sangon Biotech, Shanghai, China) were $2 \mu \mathrm{g} /$ well for SV40 MES13 cells in 6-well plates. All transfections were performed using Liposome ${ }^{\mathrm{TM}}$ 3000 transfection reagent (Kusatsu Takayama, Japan). After transfection for $48 \mathrm{~h}$, follow-up experiments were carried out with SV40 MES13 cells.

\section{RT-qPCR Analysis}

Total RNA was extracted from mice nephridial tissues or SV40 MES13 cells using TIZOL reagents (Takara, Kusatsu, Japan), and the RNA concentration was measured with absorbance at a wavelength of $260 \mathrm{~nm}$. M-MLV reverse transcriptase (RNase H) kits were used for the synthesis of cDNA. RT-qPCR was done using SYBR Green PCR Master Mix (Takara, Kusatsu, Japan). mRNA testing was carried out with GAPDH as a negative control. The relative expression was calculated by using the method of $2^{-\Delta \Delta C T}$. The primers applied to this study are shown in Table 1.

\section{Western Blot}

The total protein was isolated from SV40 MES13 cells using cell lysis buffer (Beyotime, Shanghai, China). The method for Western blot is described previously [17]. All antibodies used in this study were obtained from Abcam (Cambridge, UK), including Collagen IV (Col-IV) (ab6586), Fibronectin (FN) (ab2413), Transforming growth factor- $\beta 1$ (TGF- $\beta 1$ ) (ab215715), and SOX6 proteins (ab245215). GAPDH (ab9485) served as the internal reference. The light density of protein bands was quantified with Image J Software.

\section{CCK-8 Assay}

Cell Counting Kit-8 (Beyotime) was applied to assess SV40 MES13 cells' viability. In detail, cells were inoculated in 96-well plates (Corning, NY, USA) and incubated with $25 \mathrm{mM} \mathrm{HG}$ for 1, $2,3,4$, and 5 days. Then, $10 \mu \mathrm{L}$ of CCK- 8 solution was added to the cell wells and incubated at $37^{\circ} \mathrm{C}$ for $2 \mathrm{~h}$. Finally, a fluorescent microplate reader was used to detect the absorbance at $450 \mathrm{~nm}$ that reflects cellular vitality. 
Table 1. Primers used in this study

\begin{tabular}{ll}
\hline Primer name & Primer sequences \\
\hline F-IncRNA DIx6os1 & 5'-GTTCTATTCCAGCCATTGACCT1-3' \\
R-IncRNA DIx6os1 & 5'-AACTCCTAATTCCTCTTCCTACCT-3' \\
F-EZH2 & 5'-AAGCACAGTGCAACACCAAG-3' \\
R-EZH2 & 5'-CAGATGGTGCCAGCAATAGA-3' \\
F-SOX6 & 5'-TAAGCAACTGATGAGGTCTC-3' \\
R-SOX6 & 5'-AGGCGATGGTGTGGTAGTT-3' \\
F-TNF-a & 5'-GCCACCACGCTCTTCTGTCTAC-3' \\
R-TNF-a & 5'-GGGTCTGGGCCATAGAACTGAT-3' \\
F-IL-1 $\beta$ & 5'-ACCTTCCAGGATGAGGACATGA-3' \\
R-IL-1 $\beta$ & 5'-CTAATGGGAACGTCACACACCA-3' \\
F-IL-6 & 5'-CACATGTCTCTGGGAAATCG-3' \\
R-IL-6 & 5'-TTGTATCTCTGGAAGTTTCAGATTGTT-3' \\
F-U6 & 5'-CTCGCTCGGCAGCACA-3' \\
R-U6 & 5'-AACGCTTCACGAATTGCGT-3' \\
F-GAPDH & 5'-CTGATGCCCCCATGTTCGT-3' \\
R-GAPDH & 5'-GCAGGAGGCATTGCTGATGA-3' \\
\hline
\end{tabular}

Flow Cytometry

Cell cycles of SV40 MES13 cells were detected by flow cytometry. In detail, $1 \times 10^{5} \mathrm{SV} 40 \mathrm{MES} 13$ cells were cultured in 12 -well plates with serum-free DMEM for $24 \mathrm{~h}$, centrifuged at $1,500 \mathrm{~g}$ for $5 \mathrm{~min}$, and washed twice with pre-cooled $1 \times$ PBS. The cells were then fixed with $70 \%$ ethanol (Solarbio), placed for $15 \mathrm{~min}$, centrifuged at $1,500 \mathrm{~g}$ for 5 min, and washed twice with pre-cooled $1 \times$ PBS. Cell distribution at each stage of the cell cycle was quantified in flow cytometers and ModiFit software (Olympus, Tokyo, Japan) after incubating for $12 \mathrm{~min}$ in the dark at $4^{\circ} \mathrm{C}$ with $1 \mathrm{mg} / \mathrm{mL}$ iodide (Sigma, St. Louis, MO, USA).

Subcellular Fractionation Analysis

Subcellular grading analysis was performed using the Paris ${ }^{\mathrm{TM}}$ Kit (Invitrogen, Waltham, MA, USA) as follows: cytoplasm and nuclei were isolated from SV40 MES13 cells using nuclei and cytoplasm extraction reagents (Beyotime). RT-qPCR was used to analyze the cytoplasm and nucleus RNA extract, where GAPDH and U6 were used as normalization controls, respectively.

\section{RIP Assay}

According to the manufacturer's instructions, the physical interaction between lncRNA Dlx6os1 and EZH2 was determined using the EZ-Magna RIP kit (Millipore, Bellerica, MA, USA). The detailed procedure was similar as previously described [18].

\section{ChIP Assay}

si-NC or si-EZH2 was amplified into the psi-CHECK2 reporter vector. Liposome ${ }^{\mathrm{TM}} 3000$ was used for co-transfection of the SOX6 promoter. Following 48-h transfection, in order to verify the binding of EZH2 to SOX6, according to the manufacturer's instructions, the EZ-Magna CHIP kit (Millipore) was used for ChIP testing. Finally, the precipitated DNA was measured via RT-qPCR.

\section{Statistical Analysis}

The average \pm standard deviation represents data from 3 independent experiments. GraphPad Prism 7.0 Software (GraphPad Software, Inc.) was used for statistical analysis of all data. The comparison between 2 groups was based on the $t$ test or single-factor ANOVA, and the comparison between multiple groups was based on the Tukey post-test. When the $p$ value is $<0.05$, the difference is statistically significant.

\section{Results}

\section{LncRNA Dlx6os1 Was Highly Expressed in DN Mice} and HG-Induced SV40 MES13 Cells

First, we analyzed the body weight, content of urine microalbumin and blood glucose, and accumulation of extracellular matrix of mice. Results showed that the body weight of $\mathrm{db} / \mathrm{db} \mathrm{DN}$ mice was increased significantly (Fig. 1a). Consistently, content of urine microalbumin and blood glucose was significantly upregulated in $\mathrm{db} / \mathrm{db}$ DN mice serum (Fig. 1b, c). PAS staining analysis indicated that there was obvious accumulation of extracellular matrix in $\mathrm{db} / \mathrm{db} \mathrm{DN}$ mice (Fig. 1d). RT-qPCR analysis revealed that lncRNA Dlx6os1 was highly expressed in DN mice and HG-induced SV40 MES13 cells (Fig. 1e, f). These results indicated that the highly expressed lncRNA Dlx6os1 may be involved in the DN process.

\section{LncRNA Dlx6os1 Knockdown Inhibits HG-Induced \\ SV40 MES13 Cell Proliferation, Fibrosis, and \\ Inflammatory Cytokine Release}

Next, we analyzed the effect of lncRNA Dlx6os1 on HG-induced SV40 MES13 cell proliferation, fibrosis, and inflammatory cytokine release. RT-qPCR analysis showed that lncRNA Dlx6os1 level was decreased in HG-induced SV40 MES13 cells, following si-Dlx6os1 transfection (Fig. 2a). CCK-8 analysis indicated that lncRNA Dlx6os1 knockdown antagonized SV40 MES13 cell proliferation induced by HG (Fig. 2b). Consistently, lncRNA Dlx6os1 knockdown slowed down the process of SV40 MES13 cell cycle accelerated by HG (Fig. 2c, d). RT-qPCR analysis indicated that lncRNA Dlx6os1 knockdown inhibited the release of inflammatory cytokines, including TNF- $\alpha$, IL- $1 \beta$, and IL-6 induced by HG (Fig. 2e). Western blot analysis showed that lncRNA Dlx6os1 knockdown inhibits the levels of fibrosis-related proteins, including Col-IV, FN, and TGF- $\beta 1$ proteins induced by HG (Fig. 2f). Collectively, lncRNA Dlx6os1 knockdown inhibits HG-induced SV40 MES13 cell proliferation, fibrosis, and inflammatory cytokine release.

\section{LncRNA Dlx6os1 Inhibits SOX6 Expression by}

Recruiting EZH2 in HG-SV40 MES13 Cells

Subsequently, we analyzed the specific mechanism by which lncRNA Dlx6os1 regulates HG-induced SV40 


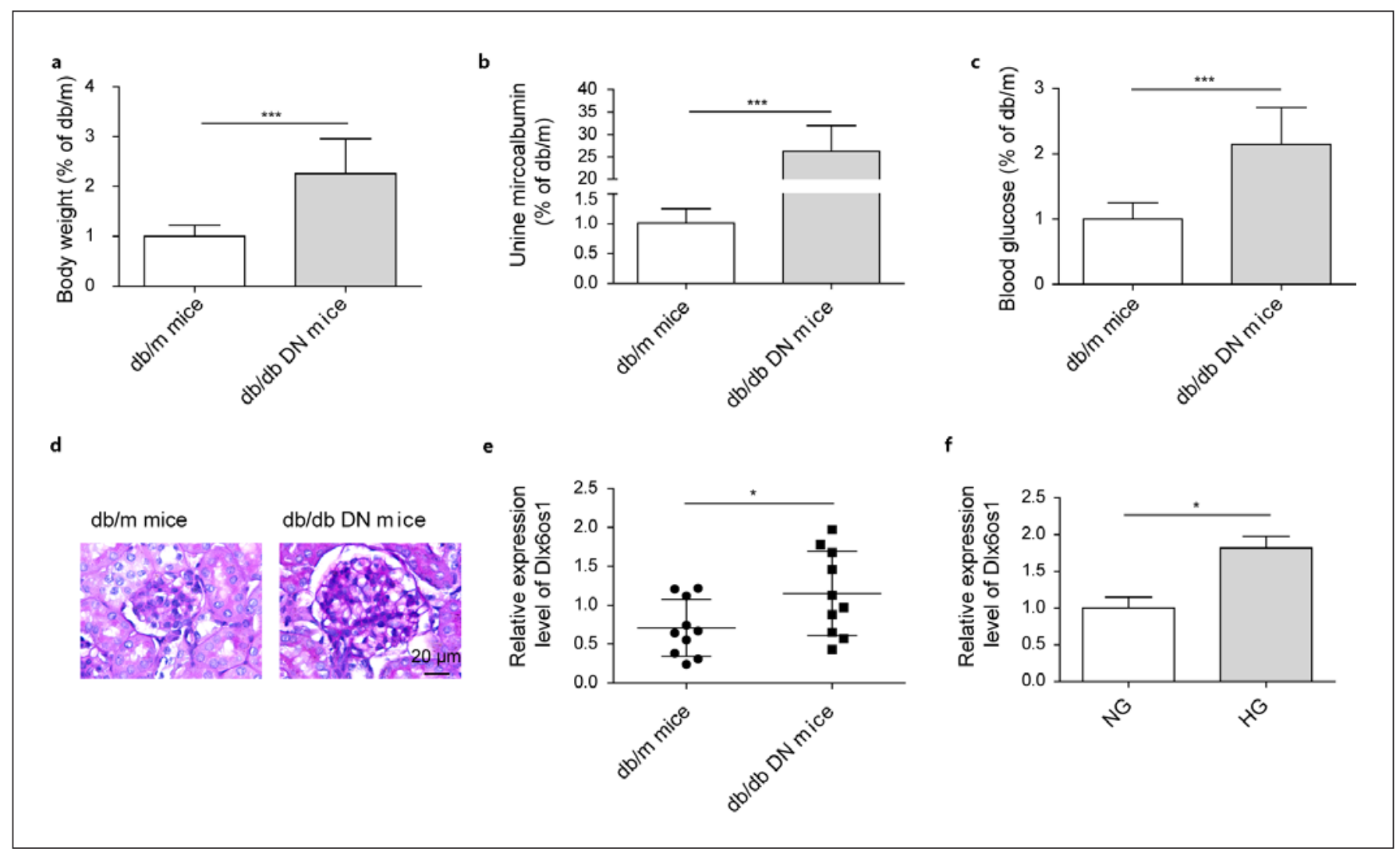

Fig. 1. LncRNA Dlx6os1 was highly expressed in DN mice and HG-induced SV40 MES13 cells. a Statistical analysis of body weight of mice. $\mathbf{b}$ ELISA was performed to detect the level of urine microalbumin in serum. c Analysis of blood glucose concentration in serum. d PAS staining was performed to analyze the mouse extracellular matrix. e-f RT-qPCR was performed to analyze lncRNA Dlx6os1 level in DN mice and HG-induced SV40 MES13 cells. ${ }^{*} p<0.05,{ }^{* *} p<0.01,{ }^{* * *} p<0.001$.

MES13 cell proliferation, fibrosis, and inflammatory cytokine release. Results indicated that lncRNA Dlx6os1 was highly expressed in the nucleus and slightly expressed in the cytoplasm of SV 40 MES13 cells, and there was a physical binding site between lncRNA Dlx6os1 and EZH2 (Fig. 3a, b). SOX6 expression was inhibited in HG-induced SV40 MES13 cells, whereas it was upregulated by lncRNA Dlx6os1 knockdown (Fig. 3c-e). Furthermore, siEZH2 was successfully transfected in SV40 MES13 cells to decrease the expression of EZH2 (online suppl. Fig. S1; for all online suppl. material, see www.karger.com/doi/10.1159/000520490). EZH2 knockdown upregulated SOX6 expression in HG-induced SV40 MES13 cells (Fig. 3f, g). EZH2 directly bound to the SOX6 promoter region, and lncRNA Dlx6os1 knockdown significantly reduced the binding activity of the SOX6 promoter (Fig. $3 \mathrm{~h}, \mathrm{i}$ ). These results suggest that lncRNA Dlx6os1 inhibits SOX6 expression by recruiting EZH2.
SOX6 Mediates the Effects of LncRNA Dlx6os1 on Proliferation, Fibrosis, and Inflammatory Factor Release of HG-Induced SV40 MES13 Cells

Finally, we analyzed the role of SOX6 in the regulation of lncRNA Dlx6os1 on proliferation, fibrosis, and inflammatory factor release of HG-induced SV40 MES13 cells. First, siSOX6 was successfully transfected in SV40 MES13 cells to decrease the expression of SOX6 (online suppl. Fig. S2). And, SOX6 knockdown promoted HGinduced SV40 MES13 cell proliferation, whereas the promoted effect was antagonized by lncRNA Dlx6os1 knockdown (Fig. 4a). Consistently, the cell cycle of HGinduced SV40 MES13 cells was accelerated by SOX6 knockdown, which was antagonized by lncRNA Dlx6os 1 knockdown (Fig. 4b, c). Furthermore, SOX6 knockdown increased the levels of inflammatory cytokines, including TNF- $\alpha$, IL- $1 \beta$, and IL- 6 , and fibrosis-associated proteins, including Col-IV, FN, and TGF- $\beta 1$, in HG-induced SV 40 


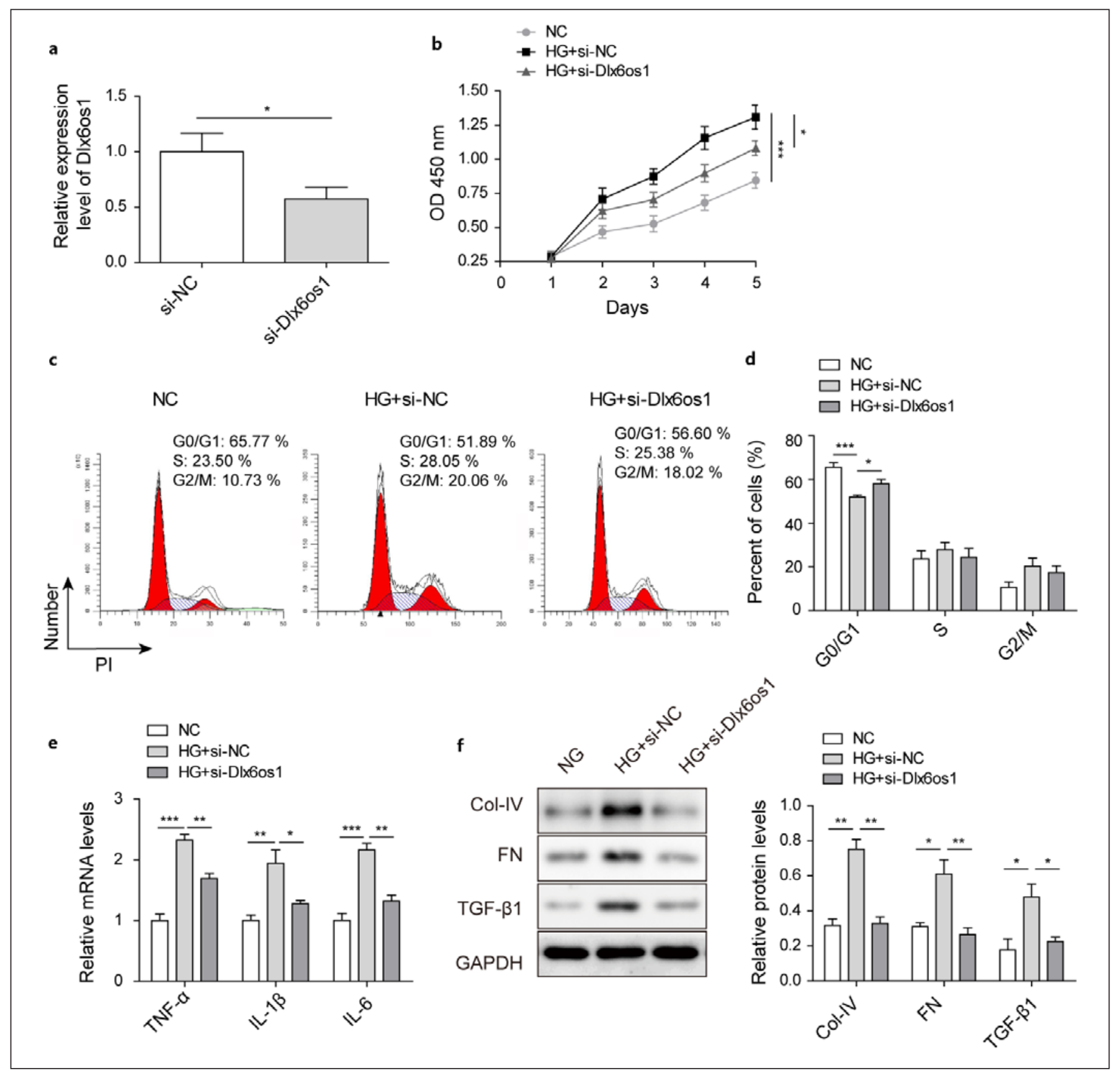

Fig. 2. LncRNA Dlx6os1 knockdown inhibited HG-induced SV40 MES13 cell proliferation, fibrosis, and inflammatory cytokine release. a RT-qPCR was performed to assess lncRNA Dlx6os1 level. b CCK-8 assay was carried out to evaluate SV40 MES13 cell proliferation. c-d Flow cytometry was performed to analyze the cell cycle of SV40 MES13 cells. e RT-qPCR was performed to detect the levels of TNF- $\alpha$, IL-1 $\beta$, and IL- 6 . f Western blot was conducted to measure the levels of Col-IV, FN, and TGF- $\beta 1 .{ }^{*} p<0.05,{ }^{* *} p<0.01,{ }^{* * *} p<0.001$.

MES13 cells, while lncRNA Dlx6os1 knockdown antagonized the promoted effect (Fig. 4d, e). Collectively, lncRNA Dlx6os1 may accelerate the $\mathrm{DN}$ process by regulating SOX6 expression.

The Effect of LncRNA Dlx6os1 on Diabetic Nephropathy

\section{Discussion}

$\mathrm{DN}$ is a vital complication of diabetes and is becoming the main cause of morbidity worldwide [19]. In this study, 


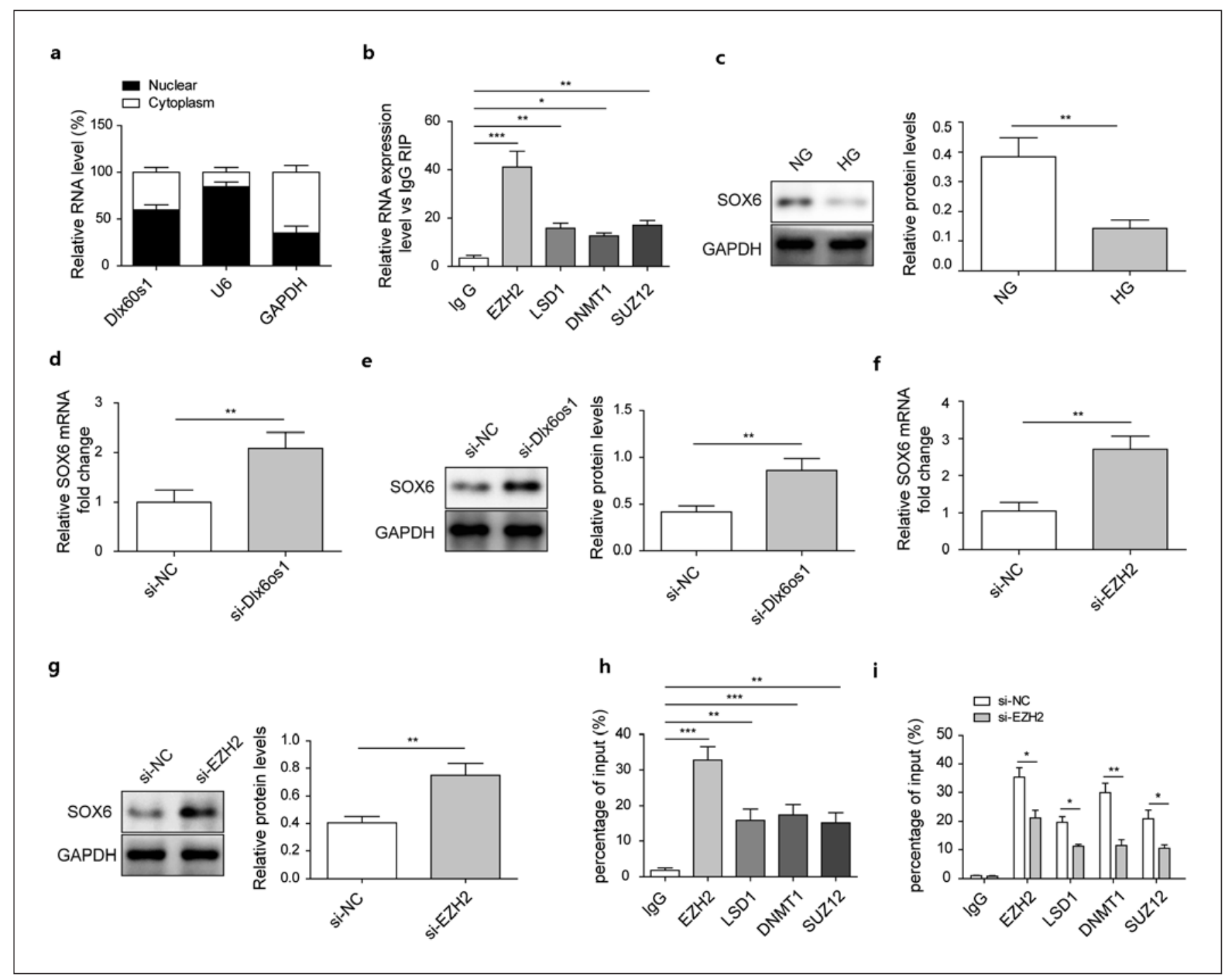

Fig. 3. LncRNA Dlx6os1 inhibits SOX6 expression by recruiting EZH2 in SV40 MES13 cells. a RT-qPCR was performed to assess lncRNA Dlx6os1 level in the cytoplasm and nucleus of SV40 MES13 cells. b RIP assay was carried out to confirm the interaction between lncRNA Dlx6os1 and EZH2. c-g The level of SOX6 in HG-induced SV40 MES13 cells. $\mathbf{h}-\mathbf{i}$ ChIP-qPCR was performed to confirm the interaction between EZH2 and SOX6 promoter. ${ }^{*} p<0.05,{ }^{* *} p<0.01,{ }^{* * *} p<0.001$.

we explored the specific mechanism by which lncRNA Dlx6os1 regulates the progression of DN. Our findings showed that lncRNA Dlx6os1 accelerates the progression of DN by epigenetically repressing SOX6 via recruiting $\mathrm{EZH} 2$, providing new insights into the development of DN treatment strategies.

It has been reported that lncRNA participates in the regulation of inflammation and cell proliferation in DN. Yi et al. [20] reported that lncRNA Gm4419 inhibits inflammation, fibrosis, and proliferation of mesangial cells via the NF- $\mathrm{B} / \mathrm{NLRP} 3$ pathway in DN. Zhang et al. [5] revealed that lncRNA Rpph1 accelerates inflammation and proliferation of neutrophils through interaction with Gal-3 in DN. LncRNA Dlx6os1, also known as EVF2, is located on chromosome 6: 68201896871592. Research has shown that inhibition of lncRNA Dlx6os1 alleviates cell proliferation and fibrosis and accelerates apoptosis in DN [12]. In this study, our results showed that lncRNA Dlx6os1 was highly expressed in DN mice and HG-induced SV40 MES13 cells, indicating lncRNA Dlx6os1 plays a positive role in $\mathrm{DN}$ progression which is consistent with previous 


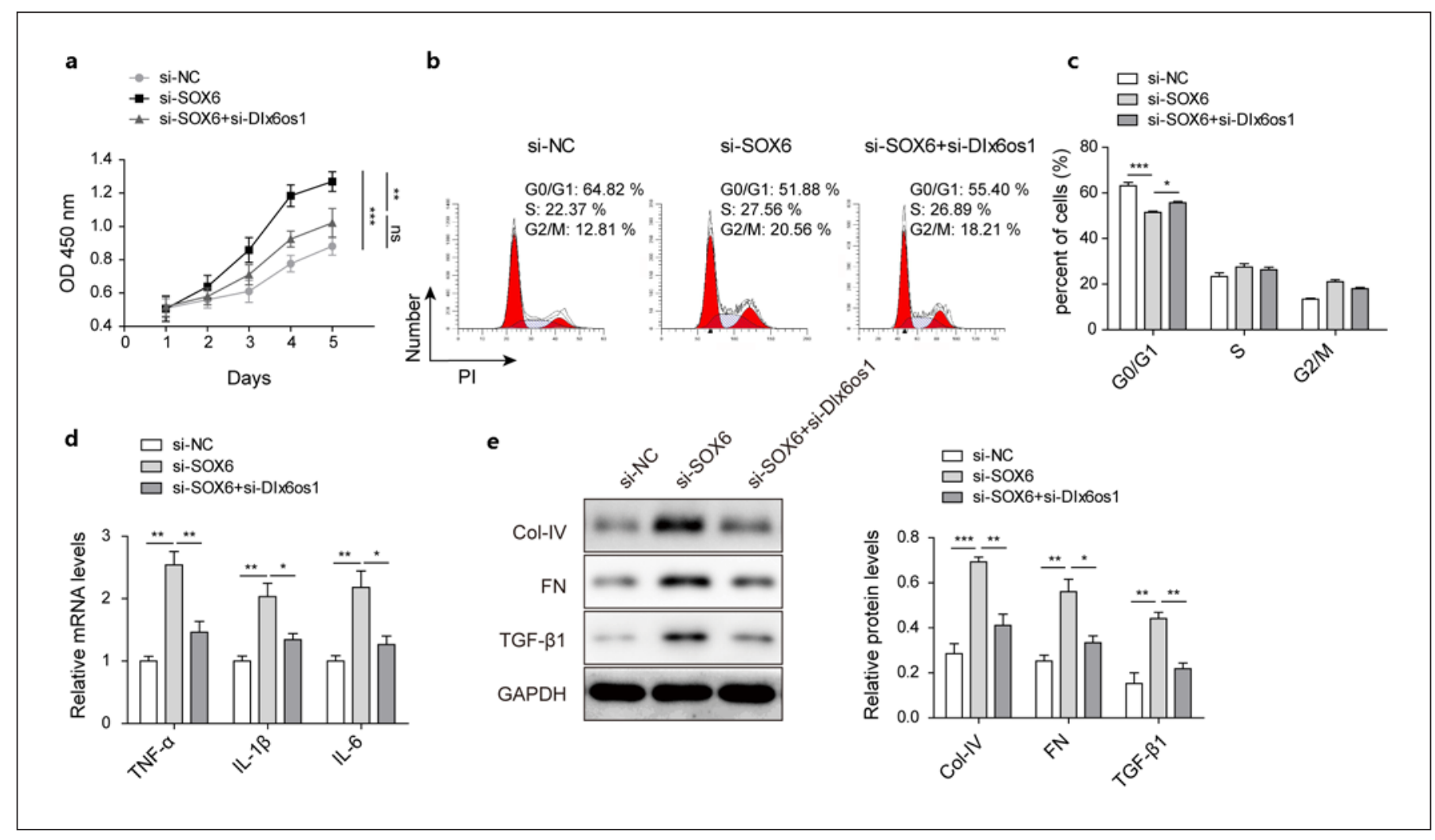

Fig. 4. SOX6 mediates the effects of lncRNA Dlx6os1 on proliferation, fibrosis, and inflammatory factor release of HG-induced SV40 MES13 cells. a CCK-8 assay was performed to assess HGinduced SV40 MES13 cell proliferation. b-c Flow cytometry was performed to analyze the cell cycle of SV40 MES13 cells. d RT-qPCR was carried out to assess the levels of inflammatory cytokines, including TNF- $\alpha$, IL-1 $\beta$, and IL-6. e Western blot was conducted to measure the levels of fibrosis-associated proteins, including Col-IV, FN, and TGF- $\beta 1 .{ }^{*} p<0.05,{ }^{* *} p<0.01$, $* * * p<0.001$. studies. Furthermore, functional studies showed that lncRNA Dlx6os1 knockdown inhibited HG-induced SV40 MES13 cell proliferation, fibrosis, and inflammatory cytokine release, suggesting lncRNA Dlx6os1 knockdown alleviates DN progression.

LncRNA regulates target genes' levels at transcriptional and posttranscriptional levels $[12,21]$. In this study, RIP assay analysis showed that lncRNA Dlx6os1 bound to EZH2 in the nucleus of SV40 MES13 cells, indicating there was a potential interaction between $\ln$ cRNA Dlx6os1 and EZH2 in DN. EZH2 is an important subunit of the PRC2 complex [21]. We speculated that lncRNA Dlx6os1 may recruit EZH2 to the SOX6 promoter to regulate its transcriptional level. As expected, ChIP assay confirmed that lncRNA Dlx6os1 bound to EZH2 on the SOX6 promoter. Mechanically, lncRNA Dlx6os1 inhibits SOX6 expression via recruiting EZH2 in HG-induced SV40 MES13 cells. Furthermore, functional research studies showed that lncRNA Dlx6os1 knockdown alleviated the effect of SOX6 knockdown on proliferation, fibrosis, and inflammatory factor release of HG-induced SV 40 MES13 cells, indicating SOX6 mediates the effects of IncRNA Dlx6os1 on proliferation, fibrosis, and inflammatory factor release of HG-induced SV40 MES13 cells.

In summary, our findings illuminated that lncRNA Dlx6os1 accelerates HG-induced SV40 MES13 cells proliferation, fibrosis, and inflammatory factor release by epigenetically repressing SOX6 via recruiting EZH2. Our study figured out the specific mechanism by which lncRNA Dlx6os1 regulates DN progression, indicating lncRNA Dlx6os1 may serve as a novel biomarker for prevention and treatment of DN.

\section{Acknowledgments}

We would like to give our sincere gratitude to the reviewers for their constructive comments. 


\section{Statement of Ethics}

This study protocol was reviewed and the need for approval was waived by The Second Affiliated Hospital of Nanchang University. Consent for publication is not applicable. This article does not contain any studies with human participants performed by any of the authors.

\section{Conflict of Interest Statement}

The authors declare that there are no conflicts of interest.

\section{Funding Sources}

This research did not receive any specific grant from funding agencies in the public, commercial, or not-for-profit sectors.

\section{Author Contributions}

Y.X.C. contributed to conceptualization and funding acquisition; S.Y.Z. contributed to writing - original draft; C.H. contributed to data curation and resources; C.Y.X. contributed to methodology and formal analysis; X.D.F. contributed to investigation, software, and visualization; W.P.T. contributed to project administration, supervision, validation, and writing - review and editing. All authors have read and approved the final version of this manuscript to be published.

\section{Data Availability Statement}

All data generated or analyzed during this study are included in this article. The datasets used and/or analyzed during the current study are available from the corresponding author on reasonable request.

\section{References}

1 Reidy K, Kang HM, Hostetter T, Susztak K. Molecular mechanisms of diabetic kidney disease. J Clin Invest. 2014;124(6):2333-40.

2 Keating ST, van Diepen JA, Riksen NP, ElOsta A. Epigenetics in diabetic nephropathy, immunity and metabolism. Diabetologia. 2018;61(1):6-20.

3 Tuttle KR, Bakris GL, Bilous RW, Chiang JL, de Boer IH, Goldstein-Fuchs J, et al. Diabetic kidney disease: a report from an ADA Consensus Conference. Am J Kidney Dis. 2014; 64(4):510-33.

4 Fried LF, Emanuele N, Zhang JH, Brophy M, Conner TA, Duckworth W, et al. Combined angiotensin inhibition for the treatment of diabetic nephropathy. N Engl J Med. 2013; 369(20):1892-903.

5 Zhang P, Sun Y, Peng R, Chen W, Fu X, Zhang $\mathrm{L}$, et al. Long non-coding RNA Rpph1 promotes inflammation and proliferation of mesangial cells in diabetic nephropathy via an interaction with Gal-3. Cell Death Dis. 2019; 10(7):526.

6 Kondo Y, Shinjo K, Katsushima K. Long noncoding RNAs as an epigenetic regulator in human cancers. Cancer Sci. 2017;108(10):192733.

7 Shao Y, Chen HT, Ma QR, Zhang YW, He YQ, Liu J. Long non-coding RNA PVT1 regulates glioma proliferation, invasion, and aerobic glycolysis via miR-140-5p. Eur Rev Med Pharmacol Sci. 2020;24(1):8249_ 83.
8 Chi Y, Wang D, Wang J, Yu W, Yang J. Long non-coding RNA in the pathogenesis of cancers. Cells. 2019;8(9):1015.

9 Liu X-H, Sun M, Nie F-Q, Ge Y-B, Zhang E-B, Yin D-D, et al. Lnc RNA HOTAIR functions as a competing endogenous RNA to regulate HER2 expression by sponging miR-331-3p in gastric cancer. Mol Cancer. 2014;13:92.

10 Li N, Jia T, Li YR. LncRNA NEAT1 accelerates the occurrence and development of diabetic nephropathy by sponging miR-23c. Eur Rev Med Pharmacol Sci. 2020;24(3):1325-37.

11 Cheng L, Cheng J, Peng W, Jiang X, Huang S. Long non-coding RNA Dlx6os1 serves as a potential treatment target for diabetic nephropathy via regulation of apoptosis and inflammation. Exp Ther Med. 2020;20(4):3791-7.

12 Cheng J, Cheng L, Tang Y, Li H, Peng W, Huang S. Inhibition of lncRNA Dlx6os1 decreases cell proliferation and fibrosis and increases cell apoptosis in diabetic nephropathy. Int J Clin Exp Pathol. 2018;11(7):3302-9.

13 Kiselak EA, Shen X, Song J, Gude DR, Wang J, Brody SL, et al. Transcriptional regulation of an axonemal central apparatus gene, sperm-associated antigen 6, by a SRY-related high mobility group transcription factor, SSOX5. J Biol Chem. 2010;285(40):30496-505.

14 Zhang L, Niu X, Zhang X, Zhan G, Xue X, Wang $X$, et al. SRY-related high-mobilitygroup box 6 suppresses cell proliferation and is downregulated in breast cancer. Anticancer Drugs. 2021;32(3):306-13.
15 Li Y, Xiao M, Guo F. The role of Sox6 and Netrin-1 in ovarian cancer cell growth, invasiveness, and angiogenesis. Tumour Biol. 2017; 39(5): 1010428317705508.

16 Saleem M, Barturen-Larrea P, Gomez JA. Emerging roles of Sox6 in the renal and cardiovascular system. Physiol Rep. 2020;8(22): e14604.

17 Dong SM, Cui JH, Zhang W, Zhang XW, Kou TC, Cai QC, et al. Inhibition of translation initiation factor eIF4A is required for apoptosis mediated by Microplitis bicoloratus bracovirus. Arch Insect Biochem Physiol. 2017; 96(3).

18 Liu XH, Sun M, Nie FQ, Ge YB, Zhang EB, Yin DD, et al. Lnc RNA HOTAIR functions as a competing endogenous RNA to regulate HER2 expression by sponging miR-331-3p in gastric cancer. Mol Cancer. 2014;13:92.

19 Liang S, Cai GY, Chen XM. Clinical and pathological factors associated with progression of diabetic nephropathy. Nephrology. 2017; 22(Suppl 4):14-9.

20 Yi H, Peng R, Zhang LY, Sun Y, Peng HM, Liu HD, et al. LincRNA-Gm4419 knockdown ameliorates NF- $\mathrm{KB} / \mathrm{NLRP} 3$ inflammasomemediated inflammation in diabetic nephropathy. Cell Death Dis. 2017;8(2):e2583.

21 LiZ, YuZ, Meng X, Yu P. LncRNA LINC00968 accelerates the proliferation and fibrosis of diabetic nephropathy by epigenetically repressing p21 via recruiting EZH2. Biochem Biophys Res Commun. 2018;504(2):499-504. 